\title{
Observational learning of a lever pressing response'
}

JOHN A. CORSON

MCGILL UNIVERSITY

Eighteen rats were taught to press a lever for food using either an observational technique or a shaping technique. The median number of sessions taken to learn was smaller when the observational technique was used.

Two systems are widely used in teaching a rat to press a lever for food, in one the experimenter shapes the behavior and in the other he simply puts the hungry animal in the box and waits for him to learn spontaneously. The problems with shaping include differences in skill and technique as well as the change in the experimenter's performance as he becomes fatigued. On the other hand the problem noticed in this laboratory with the technique of leaving the animal alone is that some will not learn at all (e.g., 6 out of 20 had not learned after $1590 \mathrm{~min}$. sessions).

Since rats learn to imitate or follow other rats in mazes (Church, 1957; Stimbert et al, 1966) and since higher animals are capable of observational learning (Herbert \& Harsh, 1944; Darby \& Riopelle, 1959), an attempt was made to use an observational technique in teaching rats to press a lever.

Method

The Ss in this experiment were 18 naive male hooded rats weighing 200 to $225 \mathrm{gm}$. Ss were maintained on a 22-hr. food deprivation schedule. The apparatus was a standard operant conditioning box enclosed in a sound resistant case (Foringer small animal test chamber). A lever and pellet receptacle protruded from one side of the box. The apparatus was set to deliver one pellet for each press of the lever. SB were handled for 15 min. each day during the first three days in the laboratory. At the beginning of the first experimental day $S$ was placed in the conditioning box with a similarly deprived sophisticated lever presser (an animal previously trained to press at least 75 times per $15 \mathrm{~min}$. session). Following $15 \mathrm{~min}$. of exposure to the pressing and eating of the sophisticated demonstrator rat, the $\mathrm{S}$ was tested alone for $15 \mathrm{~min}$.

These two situations (observation and test) were repeated three times per day until $S$ was pressing at the rate of 50 or more times in a 15 min. test session. Thus, the time spent in the apparatus on any day totalled no more than $90 \mathrm{~min}$. Twelve rats were trained in this way, each paired with one of five sophisticated rats throughout. The other six rats were trained to press the lever using a shaping technique. Since instructions for shaping were not found in the literature, the experimenter operated the food magazine in an attempt to train $S$ successively to eat from the pellet receptacle, to approach the receptacle after hearing the magazine operate, to approach the lever in order to make the magazine operate and finally to press the lever in order to make the magazine operate. These animals were shaped in $20 \mathrm{~min}$. daily sessions untll they had reached the criterion of $\mathbf{5 0}$ presses in a $15 \mathrm{~min}$. period. The shaping sessions were limited to $20 \mathrm{~min}$. because of experimenter fatigue and an apparent stereotyping of subject behavior which had been observed in longer pilot sessions. Results and Discussion

The number of sessions taken by animals in both groups is presented in Table 1. The mean and median numbers of test sessions (or cycles) for the observational group were 4.4 and 2 , respectively. The mean and median numbers of sessions taken by the shaped Ss were 6.6 and 6.5 , respectively.

Use of the median test in combination with Fisher's exact probability test (Ferguson, 1959) to compare the se groups shows that the observational group learned in fewer test sessions $(p=.0045)$. No stable differences were observed in the rate of learning of naive rats paired with particular sophisticated rats.

While there was no difference in the total time to criterion (132.5 $\mathrm{min}$. $8 \mathrm{~s} .133 .3 \mathrm{~min}$.) there are important advantages to the observational technique. For example, the number of animals which can be taught in a given period is limited by the number of available conditioning boxes and sophisticated rats rather than by the number of experimenters. On the other hand, the greater variabllity of the results in the observational group may represent a distinct disadvantage in some experiments.

It is likely that many variables could influence the rate of learning in situations described here. These

Table 1. Number of Test Sessions to Criterion

\begin{tabular}{cccc} 
S & $\begin{array}{c}\text { Observational } \\
\text { Sessions }\end{array}$ & S\# & Shaped \\
\hline 1 & 13 & 13 & 6 \\
2 & 1 & 14 & 8 \\
3 & 2 & 15 & 6 \\
4 & 4 & 16 & 8 \\
5 & 1 & 17 & 5 \\
6 & 2 & 18 & 7 \\
7 & 12 & & \\
8 & 3 & & \\
9 & 10 & & \\
10 & 2 & & \\
11 & 2 & & \\
12 & 1 & & \\
\hline
\end{tabular}


include variations in the apparatus such as lever pressure and location of the pellet receptacle relative to the lever, variations in duration of observation and test phases and variations in subject anxiety or adaptation as well as a variety of social interactions. Observational learning deserves further research both as an interesting and important phenomenon and as a useful experimental technique. Recent pilot studies with cats as Ss suggest that the most dramatic results in both respects will be obtained with animals higher than the rat. For example, considerable difficulty was experienced in shaping two cats to turn a wheel for milk while three other cats learned relatively quickly when an observational technique was used.

\section{References}

Church, R. M. Two procedures for the establishment of "imitative behavior." J. comp. physiol. Psychol., 1957, 50, 315-318.

Darby, C. L., \& Riopelle, A. J. Observational learning in the Rhesus monkey. J. comp. physiol. Psychol., 1959, 52, 94-98.

Ferguson, G. A. Statistical analysis in psychology and education. New York: McGraw-Hill, 1959.

Herbert, M. J., \& Harsh, C. M. Observational learning by cats. J. comp. Psychol., 1944, 37, 81-95.

Stimbert, V. E., Schaeffer, R. W., \& Grimsley, D. L. Acqui sition of an imitative response in rats. Psychon. Sci., 1966, 5, 339-340.

\section{Nofe}

1. This research was supported by Medical Research Council of Canada Grant MA 1746 and by Public Health Service Fellowship 2 F2 HD- $25,618-02 \mathrm{G}+\mathrm{D}$. 